Gynäk. Rdsch. 1979;19:I-VI

\title{
Contents, Vol. 19, 1979
}

\section{Inhaltsverzeichnis}

Nr. 1

Käser, O. et Stucki, D.: Malformations utérines et reproduction. Valeur de la métroplastie 1

Semm, K.: Die Geburtseínleitung mit PGF2 $\alpha$

Parsa, H. et Pelasseyed, A.A.: Traitement des tumeurs trophoblastiques par la chirurgie et par la chimiothérapie, seules ou associées 23

Mende, T.; Henning, K.; Wollny, G.; Gens, J. und Zotter, S.: Verteilung von Jod-markiertem Diäthylstilböstroldiphosphat nach intravenöser Injektion. Szintigraphische Darstellung von Mammatumoren im Tierexperiment $\quad 30$ Kurzfassungen von Zeitschriftenartikeln Reform des Referatenteiles der „Gynäkologischen Rundschau” 37 Alexander, H.; Herter, U.; Radzuweit, H. und Brockmann, J.: Das Verhalten des Serumimmunglobulin E beim Genitalkarzinom der Frau 38 Tchobroutsky, C.; Amiel-Tison, C.; Cedard, L.; Eschwege, E.; Rouvillois, J.L. und Tchobroutsky, G.: Die L/S-Ratio bei 132 insulinabhängigen diabetischen Schwangerschaften 39

Gitsch, E.; Schneider, W.H.F. und Spona, J.: Eine Ostrogen-Gestagen-Behandlung steigert bei amenorrhoischen Patientinnen die ovulatorische Wirksamkeit von Clomiphen 41

Hamann, B.; Sielaff, E. und Büchner, C: Endometriumabdruckzytologie mit Intrauterinpessar zur Korpuskarzinomfrüherkennung 42

Mueller-Heubach, E.; Caritis, S.N.; Edelstone, D.I. und Turner, J.H.: Die L/S-Ratio im Fruchtwasser zur Vorhersage eines RDS bei durch Diabetes mellitus komplizierten Schwangerschaften 43

Jaquet, P.; Grisoli, F.; Guibout, M.; Lissitzky, J.C. und Carayon, P.: Prolaktinsezernierende Tumoren: Endokrinologischer Status vor und nach der Operation bei 33 Frauen $\quad 45$

Griffith, R.W.;Turkali, I. und Braun, P.: Schwangerschaftsausgang nach Bromokryptintherapie $\quad 47$

Habib, Z.A.: $\alpha$-Fetoprotein im mütterlichen Serum: Wert einer antenatalen Diagnose hinsichtlich genetischer Erkrankungen in der geburtshilfhch-gynäkologischen Vorsorge 49

Hirsch, H.A.; Ledger, W.J.; Sweet, R.L. und Schoop, H.D.: Anaerobierinfektionen in der Geburtshilfe und Frauenheilkunde. Erstes Tübinger Anaerobiergespräch ... 51 Inhaltsverzeichnis

IV

Berger, C; Baumann, U.; Radakovic, D.; Ramzin, M.; Richter, R. und Schenk,W.: Die Geburt unter Periduralanästesie $\quad 54$ 
Breuker, K.H.; Winkhaus-Schindl, I. und Citoler, P.: Chromosomenanomalien bei Ehepaaren mit wiederholten Aborten 56

Herms, V. und Kubli, F.: Psychosomatische Aspekte von Schwangerschaft, Geburt und Wochenbett 58

Postgraduate Training Fischer, B. von und Rentsch, H.P.: Schwangerschaft und Porphyrie.

Fallbesprechung . 60

Nr. 2

Original arbeiten

Schaer, H.M.: Anästhesie- und Intensivbehandlungsprobleme bei der manifesten

Eklampsie $\quad 65$

Hermanns, U.; Kemeter, P.; Fulmek, R.; Wolf, G.; Stöger, S.; Polak, S. und Friedrich,

F.: Die diagnostische Bewertung des Prolaktin-Befundes in der Sterilitäts- und

Hormonambulanz 77

Kurzfassungen von Zeitschriftenartikeln

Litschgi, M. und Glatthaar, E.: Primäre Dysmenorrhö und Hyperprolaktinämie .... 105 Wolf, A.S.; Musch, K.; Fehm, H.L. und Lauritzen, C: Untersuchungen zur Hyper-

emesis gravidarum 106

Barlow, D.: Kondom und Gonorrhö 108

Jones, H.W. und Rock, J.A.: Tubenanastomose nach chirurgischer Sterilisation .... 109

Richardson, J.A.; Sutherland, I.A. und Allen, D.W.: Ein 'Cervimeter' fúr kontinuier-

liche Messung der Muttermundweite unter der Geburt - Vorläufige Ergebnisse . 111 Nillius, S.J.; Bergquist, C. und Wide, L.: Ovulationshemmung durch Langzeitverab-

reichung von stimulierenden LRH-analogen Substanzen - eine neue Möglichkeit

zur Geburtenkontrolle? 112

Tatra, G. und Schreiber, V.: Korrelation der mütterlichen Serumkonzentration von

HPL und SP-1 in der zweiten Schwangerschaftshälfte 114

Hastwell, G.B.: Beschleunigte Blutgerinnung durch den Thromboplastingehalt im

Fruchtwasser: eine Methode zur Bestimmung der fetalen Reife 115

Blumenfeld, T.A.; Stark, R.I.; James, L.S.; George, J.D.; Dyrenfurth, I.; Freda, V.J.

und Shinitzky, M.: Bestimmung der fetalen Lungenreife mit Hilfe der Fluores-

zenzpolarisation des Fruchtwassers 116

Piver, M.S.; Barlow, J.J. und Xynos, F.P.: Adriamycin allein oder in Kombination bei

100 Patientinnen mit Karzinom der Zervix oder Vagina 118

Peyton, F.W.; Peyton, R.R.; Anderson, V.L. und Pavnica, P.: Die Bedeutung der

Verschorfungsbehandlung zur Erhaltung einer gesunden Zervix 119

Inhaltsverzeichnis

$\mathrm{V}$

Chien-Tien Hsu und I-Sen Liu: Operative Behandlung des Zervixkarzinoms (Ergebnisse

des Provincial Taipei Hospital) zwischen 1955 und 1958

Schlosser, H.: Erfahrungsbericht über 495 Konisationen

Pirofsky, B.: Immunologic Aspects of Malignancy

121

123

Freedman, R.S.; Wharton, J.T.; Rutledge, F. und Sinkovics, J.G.: Der Transferfaktor

und seine Anwendungsmöglichkeiten in der Gynäkologie

127

Nr. 3

Originalarbeiten

Kucera, H.; Reinold, E. und Schönswetter, P.: Die perinatale Problematik des zweiten 
Zwillings $\quad 129$

Morf, P. Das hellzellige Ovarialkarzinom 139

Ehrler, P.: Der ,, to tale" Tubenverschluss und die ,,intramurale" Tubenanastomose.

Ampullouterine und isthmouterine Anastomose als Alternative zur Implantation:

Sammelstatistik 1962-1978 145

Holzer, E.: Tumorbedingte Uterusinversion. Fallbericht , 150

Da Rugna, D.: Pruritus vulvae 156

Kurzfassungen von Zeitschriftenartikeln

Kauraniemi, T.; Räsänen-Virtanen, U. und Hakama, M.: Das Risiko für ein Zervixkar-

zinom nach Elektrokoagulation 165

Jorde, A.; Hammann, B.; Deicke, P. und Belling, K.H.: Ein kubisches Stützpessar im

Rahmen der Behandlung 166

Litschgi, M. und Schenk, W.: Mögliche Spätkomplikationen nach Periduralanästhesie . 168

Koenig, U.D. und Müller, N.: Humorale Immunreaktionen bei Patientinnen mit einem

Zervixkarzinom: Lymphozytotoxische Antikörper 170

Kreibich, H. und Ehrig, E.: Der Einfluss der Interruptio auf die spätere Fertilität unter

besonderer Berücksichtigung des Abortgeschehens 171

Mitzkat, H.J. und Schönhöfer, P.S.: Geburtshilflich indizierte Pharmaka mit Einfluss

auf den Kohlenhydratstoffwechsel 172

Seifert, B.; Liedtke, M.-P. und Bettzieche, H.: Untersuchungen zur Funktion des

Corpus luteum in der mittleren Lutealphase nach Behandlung mit Prostaglandinen 174 Gerhard,

I. und Runnebaum, B.: Aussagewert von HCG-, HPL-, Progesteron- und

Östriolbestimmungen bei Frauen mit drohender Fehlgeburt 175

Schulze, G. und Herold, C: Komplikationen der Interruptio und ihre Auswirkungen

auf nachfolgende Schwangerschaften 176

Peyton, F.W.; Peyton, R.R.; Anderson, V.L.; Pavnica, P. und Peyton, F.W.: Die

Bedeutung der Verschorfungsbehandlung zur Erhaltung einer gesunden Zervix . 177 Lehtovirta,

P. und Forss, M.: Die momentane Beeinflussung des intervillösen Blut-

flusses der Placenta durch Rauchen 179

Inhaltsverzeichnis VI

No. 4

Thermographie und Brustkrebs: Diagnose, Prognose, Überwachung

Der aktuelle Platz der Thermographie in der Senologie

M. Gautherie, Strasbourg, und CM. Gros, Bern 181

Kurzfassungen von Zeitschriftenartikeln

Lafaye, C. und Aubert, B.: Lokale Progesterontherapie bei benignen Brustver-

änderungen 228

Martin, P.M.; Rolland, P.H.; Gammerre, M.; Serment, H. und Toga, M.: Östrogen-und

Progesteronrezeptoren bei normalen und neoplastischem Endometrium: Korrela-

tionen zwischen Rezeptorgehalt, Histologie und klinischem Verlauf unter Gesta-

gentherapie 229

Kunz, S.: Pelvine Entzündungen bei Intrauterinpessaren 230

Guillebaud, J.; Bamett, M.D. und Gordon, Y.B.: Plasmaferritinspiegel als Massstab fúr

einen Eisenmangel bei Frauen mit Intrauterinpessaren 233

Shaw, R.W.: Veränderte Reaktion auf LH-RH nach Ostrogenvorbehandlung bei Frauen mit Amenorrhö 234 
Klittich, L.; Lehr, H.J.; Schmidt-Elmendorff, H. und Steyer, M.: Beeinflussung des Grössenwachstums grosswüchsiger Mädchen durch Östrogene 236 Brandau, H.: LH-Bestimmungen im Urin mit dem HI-Gonavis-Test zur Ovulationsvorhersage 238

Autorenindex 240

Supplementum 1

Vaginale Hysterektomie und Inkontinenz-Operationen

Symposium, Stuttgart, 18. November 1978

Supplementum 2

Schweizerische Gesellschaft für Gynäkologie

Bericht über die Jahresversammlung, 28. bis 30. Juni 1979 in Montreux

Société Suisse de Gynécologie

Compte rendu de $\Gamma$ Assemblée annuelle, 28 au 30 juin 1979 à Montreux 